\title{
Control of Inhomogeneous Ensembles on The Bloch Sphere
}

\author{
Philip Owrutsky $\left.\right|^{\text {f }}$ and Navin Khaneja \\ School of Engineering \& Applied Sciences, \\ Harvard University, Cambridge, MA 0213 $\$^{\mp}$
}

(Dated: March 1, 2022)

\begin{abstract}
Finding control fields (pulse sequences) that can compensate for the dispersion in the parameters governing the evolution of a quantum system is an important problem in coherent spectroscopy and quantum information processing. The use of composite pulses for compensating for dispersion in system dynamics is widely known and applied. In this paper, we introduce new pulse elements for correcting pulse errors. These design methods are analytical and can be used to prove arbitrarily good robust performance. Furthermore, the time-to-compensation is superior to existing Fourier Synthesis Methods which is critical for minimizing errors due to relaxation effects.
\end{abstract}

\section{INTRODUCTION}

Many applications in control of quantum systems involve controlling a large ensemble by using the same control field. In practice, the elements of the ensemble could show variation in the parameters that govern the dynamics of the system. For example, in magnetic resonance experiments, the spins of an ensemble may have large dispersion in their natural frequencies (Larmor dispersion), strength of applied rf-field (rf-inhomogeneity) and the relaxation rates of the spins 2 to name a few. In solid state NMR spectroscopy of powders, the random distribution of orientations of inter-nuclear vectors of coupled spins within an ensemble leads to a distribution of coupling strengths [13]. A canonical problem in control of quantum ensembles is to develop external excitations that can simultaneously steer the ensemble of systems with variation in their internal parameters from an initial state to a desired final state. These are called compensating pulse sequences as they can compensate for the dispersion in the system dynamics. From the standpoint of mathematical control theory, the challenge is to simultaneously steer a continuum of systems between points of interest with the same control signal. Typical applications are the design of excitation and inversion pulses in NMR spectroscopy in the presence of larmor dispersion and rf-inhomogeneity [2, 10,12] or the transfer of coherence or polarization in coupled spin ensemble with variations in the coupling strengths [14. In many cases of practical interest, one wants to find a control field that prepares the final state as some desired function of the parameter. For example, slice selective excitation and inversion pulses in magnetic resonance imaging [15 18]. The problem of designing excitations that can compensate for dispersion in the dynamics is a well studied subject in NMR spectroscopy and extensive literature exists on the subject of composite pulses that correct for dispersion in system dynamics 28]. Composite pulses have

\footnotetext{
* philip.owrutsky@gmail.com

$\dagger$ navin@eecs.harvard.edu http://hrl.harvard.edu/ navin

$\ddagger$ The work was supported by ONR 38A-1077404, AFOSR FA955005-1-0443 and AFOSR FA9550-04-1-0427
}

recently been used in quantum information processing to correct for systematic errors in single and two qubit operations 19 24].

The focus of this paper is to present novel pulse elements for compensating errors arising from uncertainties/imperfections in the pulse amplitude. The constructions presented here have the advantage that they are analytical and exhibit favorable performance compared to the existing analytical Fourier Synthesis Method [1]. Namely, a higher level of rf robustness is obtained for the same pulse length and power, which is critical for minimizing errors due to relaxation.

To fix ideas, consider an ensemble of noninteracting spin $\frac{1}{2}$ particles in a static field $B_{0}$ along the $z$ axis and a transverse rf-field, $(A(t) \cos (\phi(t)), A(t) \sin (\phi(t)))$, in the $x-y$ plane. Let $x, y, z$ represent the coordinates of the unit vector in the direction of the net magnetization vector of the ensemble. The dispersion in the amplitude of the rf-field is given by a dispersion parameter $\epsilon$ such that $A(t)=\epsilon A_{0}(t)$ where $\epsilon \in[1-\delta, 1+\delta]$, for $\delta>0$. The Bloch equations for the ensemble take the form

$$
\frac{d}{d t}\left[\begin{array}{l}
x \\
y \\
z
\end{array}\right]=\left[\begin{array}{ccc}
0 & -\omega & \epsilon u(t) \\
\omega & 0 & -\epsilon v(t) \\
-\epsilon u(t) & \epsilon v(t) & 0
\end{array}\right]\left[\begin{array}{l}
x \\
y \\
z
\end{array}\right],
$$

where

$$
(u(t), v(t))=\gamma\left(A_{0}(t) \cos (\phi(t)), A_{0}(t) \sin (\phi(t))\right) .
$$

Let $X(t)$ denote the unit vector $(x(t), y(t), z(t))$. Consider now the problem of designing controls $u(t)$ and $v(t)$ that simultaneously steer an ensemble of such systems with dispersion in the strength of their rf-field from an initial state $X(0)=(0,0,1)$ to a final state $X_{F}=(1,0,0)$ 10. This problem raises interesting questions about controllability, i.e., showing that in spite of bounds on the strength of the rf-field, $\sqrt{u^{2}(t)+v^{2}(t)} \leq A_{\max }$, there exist excitations $(u(t), v(t))$, which simultaneously steer all the systems with dispersion in $\epsilon$, to a ball of desired radius $r$ around the final state $(1,0,0)$ in a finite time (which may depend on $A_{\max }, B, \delta$, and $r$ ). Besides steering the ensemble between two points, we can ask for a control field that steers an initial distribution of the ensemble to a final distribution, i.e., different elements of 
the ensemble now have different initial and final states depending on the value of the their dispersion parameter $\epsilon$. The initial and final state of the ensemble are described by functions $X_{0}(\epsilon)$ and $X_{F}(\epsilon)$ respectively. Consider the problem of steering an initial distribution $X_{0}(\epsilon)$ to within a desired distance $r$ of a target function $X_{F}(\epsilon)$ by appropriate choice of controls in equation (1). We use the L2 norm as our error metric

$$
E^{2}=\int_{1-\delta}^{1+\delta}\left\|X_{F}(\epsilon)-X_{\text {target }}(\epsilon)\right\|^{2} d \epsilon .
$$

If a system with dispersion in its parameters can be steered between states that have dependency on the dispersion parameter arbitrarily well, then we say that the system is ensemble controllable with respect to those parameters.

This paper is organized as follows. In the following section, we introduce the key ideas and through examples, highlight the role of non-commutativity in the design of a compensating control. We present novel pulse elements that compensate for inhomogeneity or uncertainty in the amplitude of the rf-field. The presented method extends known techniques for pulse sequences that are robust to rf inhomogeneity. The methods presented may also find applications in design of anisotropy compensating pulse design or in solid state NMR experiments [9].

\section{NOVEL PULSE ELEMENTS FOR COMPENSATING RF-INHOMOGENEITY}

\section{A. Fourier Synthesis Technique}

\section{Example 1: Main Concept}

To fix ideas, we begin by considering the Bloch equations in a rotating frame with only rf-inhomogeneity and no Larmor dispersion.

$$
\dot{X}=\epsilon\left(u(t) \Omega_{y}+v(t) \Omega_{x}\right) X,
$$

where

$\Omega_{x}=\left[\begin{array}{ccc}0 & 0 & 0 \\ 0 & 0 & -1 \\ 0 & 1 & 0\end{array}\right], \Omega_{y}=\left[\begin{array}{ccc}0 & 0 & 1 \\ 0 & 0 & 0 \\ -1 & 0 & 0\end{array}\right], \Omega_{z}=\left[\begin{array}{ccc}0 & -1 & 0 \\ 1 & 0 & 0 \\ 0 & 0 & 0\end{array}\right]$

are the generators of rotation around $x, y$ and $z$ axis, respectively.

We define the pulse elements [1]

$$
\begin{aligned}
& U_{1}\left(\beta_{k}\right)=\exp \left(-\gamma_{k} \Omega_{x} \epsilon\right) \exp \left(\frac{\beta_{k}}{2} \Omega_{y} \epsilon\right) \exp \left(\gamma_{k} \Omega_{x} \epsilon\right), \\
& U_{2}\left(\beta_{k}\right)=\exp \left(\gamma_{k} \Omega_{x} \epsilon\right) \exp \left(\frac{\beta_{k}}{2} \Omega_{y} \epsilon\right) \exp \left(-\gamma_{k} \Omega_{x} \epsilon\right) .
\end{aligned}
$$

which correspond to directly accessible evolutions.

For suitably small $\beta_{k}$, we have

$$
V_{k}=U_{2} U_{1} \sim \exp \left(\epsilon \beta_{k} \Omega_{y} \cos \left(\gamma_{k} \epsilon\right)\right) .
$$

To effect a larger amplitude rotation, we consider the sequence of transformations

$$
H_{1} \equiv \prod_{k}\left(V_{k}\right)^{n_{k}} \sim \exp \left(\epsilon \sum_{k} \alpha_{k} \cos \left(\gamma_{k} \epsilon\right) \Omega_{y}\right),
$$

where $\alpha_{k}=n \beta_{k}$. In practice, $\beta_{k}<\frac{\pi}{10}$ is suitably small and results in an error that is less than $1 \%$ in the L2 sense in (6). Now, the coefficients $\alpha_{k}$ can be chosen so that

$$
\sum_{k} \alpha_{k} \cos \left(\gamma_{k} \epsilon\right) \approx \frac{\theta}{\epsilon}
$$

for $1-\delta \leq \epsilon \leq 1+\delta$, with $0<\delta<1$.

Therefore,

$$
H_{1} \sim \exp \left(\theta \Omega_{y}\right)
$$

approximately independent of $\epsilon$. The dependence on $\epsilon$ can be made arbitrarily small by increasing the pulse length and extending the number of terms in the summation leading to pulses that are immune to dispersions in the rf amplitude as claimed.

\section{B. Modified Fourier Synthesis Using $\delta$ Modulation}

In this section we develop a modified Fourier Synthesis Method that will be shown to have favorable timerobustness properties to the original Fourier Synthesis technique. To this end we consider the following system

$$
\dot{Y}=\left(\epsilon u(t) \Omega_{x}+v(t) \Omega_{z}\right) Y
$$

which corresponds to a system with one pure control by way of $v(t)$ and one control with dispersion, $\epsilon u(t)$. We will apply a similar Fourier Synthesis Method (FSM) analysis on the system and show that this results in a modified Hamiltonian to that of the previous section, the advantages of which will be discussed in section III. We then show how the previous system with both controls exhibiting dispersion (3) can be transformed into (10) by an appropriate change of coordinates.

Consider the modified transformations

$$
\begin{aligned}
& \tilde{U}_{1}=\exp \left(-\gamma_{k} \epsilon \Omega_{x}\right) \exp \left(\frac{\beta_{k}}{2} \Omega_{z}\right) \exp \left(\gamma_{k} \epsilon \Omega_{x}\right) \\
& \tilde{U}_{2}=\exp \left(\gamma_{k} \epsilon \Omega_{x}\right) \exp \left(-\frac{\beta_{k}}{2} \Omega_{z}\right) \exp \left(-\gamma_{k} \epsilon \Omega_{x}\right)
\end{aligned}
$$

By again choosing $\beta_{k}$ sufficiently small, we have

$$
\tilde{V}_{k}=\tilde{U}_{2} \tilde{U}_{1} \sim \exp \left(\beta_{k} \sin \left(\gamma_{k} \epsilon\right) \Omega_{y}\right) .
$$

Applying a sequence of such transformations

$$
H_{2} \equiv \prod_{k}\left(\tilde{V}_{k}\right)^{n_{k}} \sim \exp \left(\sum_{k} \alpha_{k} \sin \left(\gamma_{k} \epsilon\right) \Omega_{y}\right)
$$



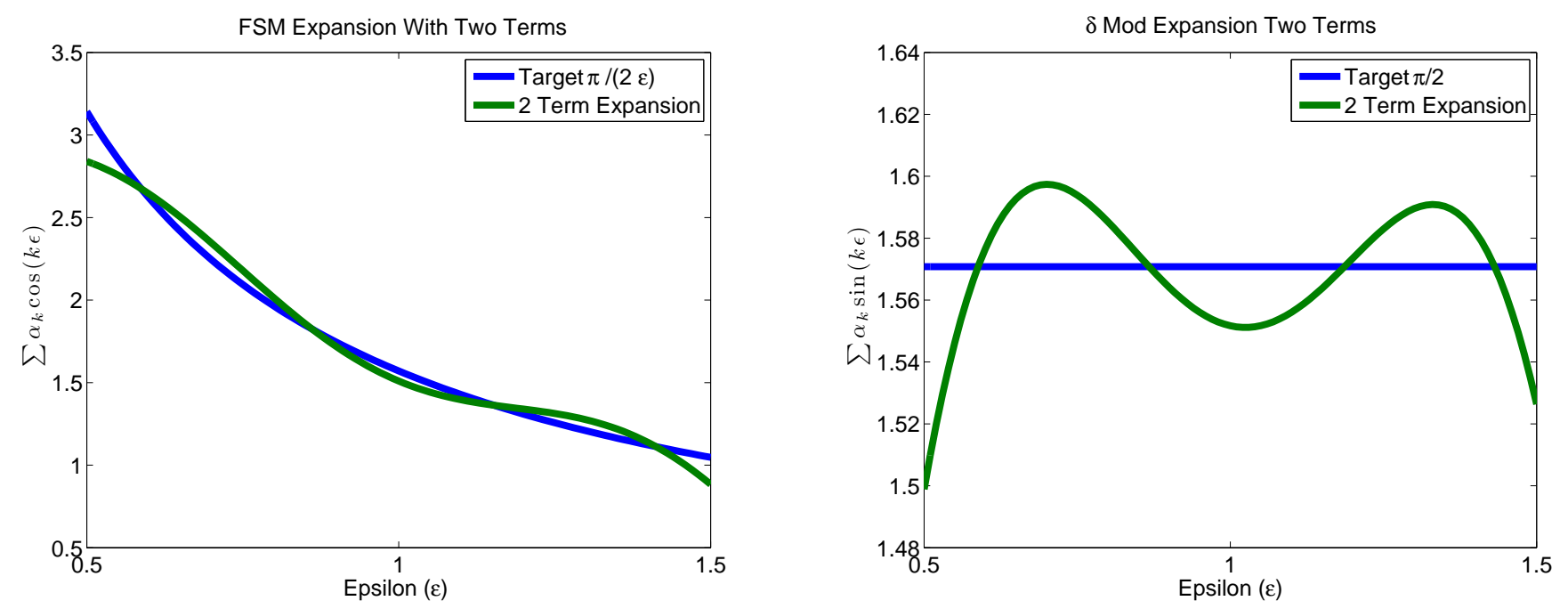

FIG. 1. Left: 2 Term approximation for $H_{1}=\frac{\pi}{2 \epsilon}$. Right: 2 Term approximation for $H_{2}=\frac{\pi}{2}$ using two terms with gradient descent for frequency selection

where again $\alpha_{k}=n \beta_{k}$ is used to control the error from the approximation in (13). Now, the coefficients $\alpha_{k}$ and $\gamma_{k}$ can be chosen so that

$$
\sum_{k} \alpha_{k} \sin \left(\gamma_{k} \epsilon\right) \approx \theta
$$

over the range of $\epsilon$ of interest $1-\delta \leq \epsilon \leq 1+\delta$ resulting in a robust rotation. We point out that (15) resembles (8), but no longer contains an $\epsilon$ factor external to the trigonometric argument and that cos has been replaced with sin.

To see how (10) can be generated from (3), we return to $(3)$

$\dot{X}=\epsilon A(\cos \underbrace{\left(\phi_{1}(t)+\phi_{2}(t)\right)}_{\phi(t)} \Omega_{x}+\sin \underbrace{\left(\phi_{1}(t)+\phi_{2}(t)\right)}_{\phi(t)} \Omega_{y}) X$

and move into the frame

$$
\begin{aligned}
Y & =\exp \left(-\phi_{2}(t) \Omega_{z}\right) X \\
\dot{Y} & =\left[\epsilon A(t)\left(\cos \phi_{1}(t) \Omega_{x}+\sin \phi_{1}(t) \Omega_{y}\right)-\dot{\phi}_{2} \Omega_{z}\right] Y(1
\end{aligned}
$$

which corresponds to 10 once the appropriate identifications are made.

This means that $\tilde{V}_{k}$ can be directly produced by implementing $\phi_{1}(t)$ as $0, \pi, \pi$ and 0 over $\Delta t$ time intervals such that $A \Delta t=\gamma_{k}$, and with $-\dot{\phi}_{2}$ a delta pulse with area $\frac{\alpha_{k}}{2}$, and $-\frac{\alpha_{k}}{2}$ at time $\Delta t$ and $3 \Delta t$ respectively in (3). As $\phi_{2}(4 \Delta t)=0, X(4 \Delta t)=Y(4 \Delta t)$ and the lab frame and $\mathrm{Y}$ frame coincide after each pulse sequence, completing the $\delta$ Modulated Pulse Design Method.

The only remaining task is selection of the amplitude and frequency coefficients which we defer until the following section where we discuss the associated performance of the algorithms.

In this paper we have focused on producing $\epsilon$-robust uniform rotations, however the reader should note that by appropriate selection of $\alpha_{k}$ in $H_{1}$ and $H_{2}$, any rotation as a function of $\epsilon$ can be produced.

\section{SIMULATIONS AND ERROR PERFORMANCE OF F. SYNTHESIS AND $\delta$ MODULATION}

$\begin{array}{lccc}\text { Heuristic } & & & \\ & \mathrm{n}=2 & \mathrm{n}=3 & \mathrm{n}=4 \\ \text { Error FSM } & 0.06831 & 0.06523 & 0.06473 \\ \text { Error } \delta \text { Mod } & 0.02012 & 0.00290 & 0.00044 \\ \text { Flip } \angle \text { FSM } & 127.120 & 187.200 & 199.600 \\ \text { Flip } \angle \delta \text { Mod } & 115.230 & 172.001 & 216.138\end{array}$

Greedy

Error FSM

Error $\delta$ Mod

Flip $\angle \mathrm{FSM}$

$\mathrm{n}=2 \quad \mathrm{n}=3 \quad \mathrm{n}=4$

0.040310 .015060 .00941

0.020290 .004220 .00247

130.497179 .062229 .101

Table 1: Performance of Fourier Synthesis and $\delta$ modulation for heuristic, greedy and gradient descent based frequency selection. In all cases, $\delta$ modulation outperforms the Fourier Synthesis method in terms of L2 Error for a given pulse duration, which is important for minimizing relaxation effects. L2 error is calculated with respect to the desired final magnetization $[1,0,0]^{\prime}$.

\section{Gradient}

Error FSM 0.073390 .018740 .00423

Error $\delta$ Mod 0.019400 .002800 .00044

Flip $\angle$ FSM 120.519225 .780347 .413

Flip $\angle \delta$ Mod 113.341170 .134216 .137 

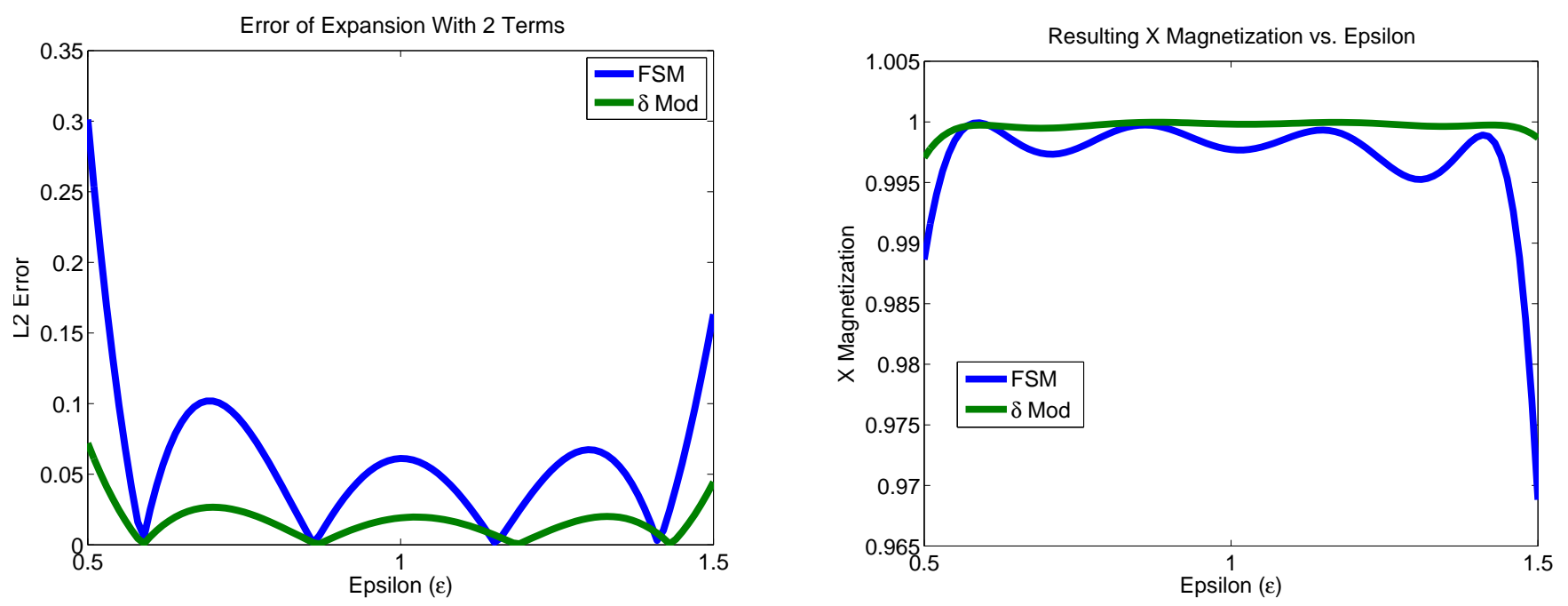

FIG. 2. Left: L2 Error with respect to the desired final magnetization $[1,0,0]^{\prime}$ for two term $H_{1}$ and $H_{2}$ as a function of the dispersion parameter $\epsilon$ where frequencies were found using gradient descent. Right: Resulting X-magnetization for the 2 term sequence for both Fourier Synthesis and $\delta$ Modulation. $\delta$ modulation has a favorable magnetization profile while requiring a shorter pulse duration.

The previous section reduced the problem of RF dispersion compensation to parameter selection, $\gamma_{k}$ and $\alpha_{k}$. Given the inhomogeneity parameter, $\epsilon \in\left[1-\delta_{0}, 1+\delta_{0}\right]$, we compute the error performance of synthesizing the effective Hamiltonians

$$
\begin{aligned}
& H_{1}=\exp \left(\epsilon \sum_{k} \alpha_{k}^{1} \cos \left(\epsilon \gamma_{k}^{1}\right) \Omega_{y}\right) \\
& H_{2}=\exp \left(\sum_{k} \alpha_{k}^{2} \sin \left(\epsilon \gamma_{k}^{2}\right) \Omega_{y}\right)
\end{aligned}
$$

to approximate

$$
H=\exp \left(\theta \Omega_{y}\right)
$$

We note that the $\left\{\alpha_{k}^{1}\right\}$ can be directly calculated given the $\left\{\gamma_{k}^{1}\right\}$ as

$$
\begin{array}{r}
\vec{\alpha}^{1}=\Phi^{-1} V ; \quad\langle f, g\rangle=\int_{1-\delta}^{1+\delta} f(\epsilon) g(\epsilon) d \epsilon \\
\Phi_{i j}=\left\langle\cos \left(\gamma_{i}^{1} \epsilon\right), \cos \left(\gamma_{j}^{1} \epsilon\right)\right\rangle ; \quad V_{i}=\left\langle\cos \left(\gamma_{i}^{1} \epsilon\right), \frac{\theta}{\epsilon}\right\rangle
\end{array}
$$

and similarly the $\left\{\alpha_{k}^{2}\right\}$ can be calculated as

$$
\begin{array}{r}
\vec{\alpha}^{2}=\Phi^{-1} V ; \quad\langle f, g\rangle=\int_{1-\delta}^{1+\delta} f(\epsilon) g(\epsilon) d \epsilon \\
\Phi_{i j}=\left\langle\sin \left(\gamma_{i}^{2} \epsilon\right), \sin \left(\gamma_{j}^{2} \epsilon\right)\right\rangle ; \quad V_{i}=\left\langle\sin \left(\gamma_{i}^{2} \epsilon\right), \theta\right\rangle
\end{array}
$$

so that the problem reduces to selecting the optimal frequencies.

We report performance for three frequency selection methods, heuristically, greedy selection and gradient descent and show that $\delta$ modulation outperforms Fourier
Synthesis Methods for all frequency selection methods. Unless stated otherwise, the notion of optimal is with respect to L2 error for a given pulse duration. L2 error is calculated with respect to the desired final magnetization $[1,0,0]$ and pulse duration is reported in total flip angle.

As a starting point, we consider the problem of selecting the optimal amplitudes given known frequencies which we will choose heuristically. As sine obtains its maximum at $\pi / 2$ and is relatively horizontal about this point, a natural selection for the frequencies in 15 is $\gamma_{k}=\frac{(2 k+1) \pi}{2}$. Similarly, selecting $\gamma_{k}$ in (8) to maximize flatness about $\epsilon=1$ corresponds to

$$
\left.\left[\frac{d}{d \epsilon} \epsilon \cos \left(\gamma_{k} \epsilon\right)\right]\right|_{\epsilon=1}=\cos \left(\gamma_{k}\right)-\gamma_{k} \sin \left(\gamma_{k}\right)=0 .
$$

Numerically solving gives the first several $\gamma_{k}=$ $[.860,3.426,6.437]$.

The amplitude coefficients $\vec{\alpha}$ were then calculated according to (20)- 23 . The comparative performance of standard FSM, $H_{1}$, to $\delta$ modulation, $H_{2}$, is tabulated in table 1 and a complete description of the pulses is given in the appendix.

An alternative algorithm is to sequentially select the frequencies employing a greedy algorithm, in which already determined frequencies are held fixed, and only the newest frequency is optimized over. Explicitly we sequentially minimize the cost functions with respect to $\gamma_{k}^{1 / 2}$

$$
\begin{gathered}
F_{1}\left(\gamma_{k}^{1}, \ldots, \gamma_{1}^{1}\right)=\int_{1-\delta}^{1+\delta}\left\|\sum \alpha_{k} \cos \left(\epsilon \gamma_{k}^{1}\right)-\frac{\theta}{\epsilon}\right\| d \epsilon \\
F_{2}\left(\gamma_{k}^{2}, \ldots, \gamma_{1}^{2}\right)=\int_{1-\delta}^{1+\delta}\left\|\sum \alpha_{k} \sin \left(\epsilon \gamma_{k}^{2}\right)-\theta\right\| d \epsilon
\end{gathered}
$$


again using (20)-23) to calculate the $\vec{\alpha}$. This was done using gradient descent and numerically calculating the necessary derivatives. Table 1 shows that the $\delta$ modulation outperforms standard FSM.

The most general method we applied (and best performing) was simultaneously optimizing $F_{1}$ and $F_{2}$ with respect to all frequencies using gradient descent, where derivatives were again calculated numerically. As with all descent schemes, there is concern that one merely obtains a local minima. Moreover, the problem of unspecified frequencies is how to project onto an overrepresented subspace which is known to have local minima. To combat such issues we chose the optimal result after numerous starting points and note that the performance exceeds the other methods and the results are displayed in table 1.

As an example we consider the resulting parameters from optimizing a two term $\delta$ modulated pulse using gradient descent

$$
\gamma^{2}=\left[88.6^{\circ}, 265.1^{\circ}\right] ; \quad \alpha^{2}=\left[105.5^{\circ}, 16.6^{\circ}\right] .
$$

These are converted into a pulse sequence by first dividing large amplitudes of $\alpha_{k}^{2}$ into repeated sequences with smaller amplitudes according to (14) using a threshold value of $9^{\circ}$, which yields the modified parameters

$$
\begin{aligned}
\gamma^{2^{\prime}} & =[\underbrace{88.6^{\circ}, \ldots, 88.6^{\circ}}_{12 \text { times }}, \underbrace{265.1^{\circ}, \ldots, 265.1^{\circ}}_{2 \text { times }}] \\
\alpha^{2^{\prime}} & =[\underbrace{8.8^{\circ}, \ldots, 8.8^{\circ}}_{12 \text { times }}, \underbrace{8.4^{\circ}, \ldots, 8.4^{\circ}}_{2 \text { times }}]
\end{aligned}
$$

Pulses are calculated as described in section II.B where pulse elements are

$$
\left[\left(\gamma_{k}\right)_{0}\left(2 \gamma_{k}\right)_{180^{\circ}-\alpha_{k} / 2}\left(\gamma_{k}\right)_{0}\right]
$$

with numbers inside the parentheses representing the flip angle and the subscripts, the phase. Applying to the modified parameters above yields the pulse sequence

$$
\left[(88.6)_{0}(177.1)_{175.6}(88.6)_{0}\right]^{\times 12}\left[(265.1)_{0}(530.1)_{175.9}(265.1)_{0}\right]^{\times 2} \text {. }
$$

The performance is displayed in figure 2 . The more terms kept in the series, the longer the sequences and overall pulse, but the more robust.

\section{GENERAL MODULATION SCHEMES}

In many ways $\delta$ modulation is the most natural choice as it has a nice correspondence with existing FSM's. However, other modulation schemes are possible and their analysis is warranted for the sake of completeness or in the event that abrupt phase adjustments in the RFfields are not available. We begin by considering linear modulation.

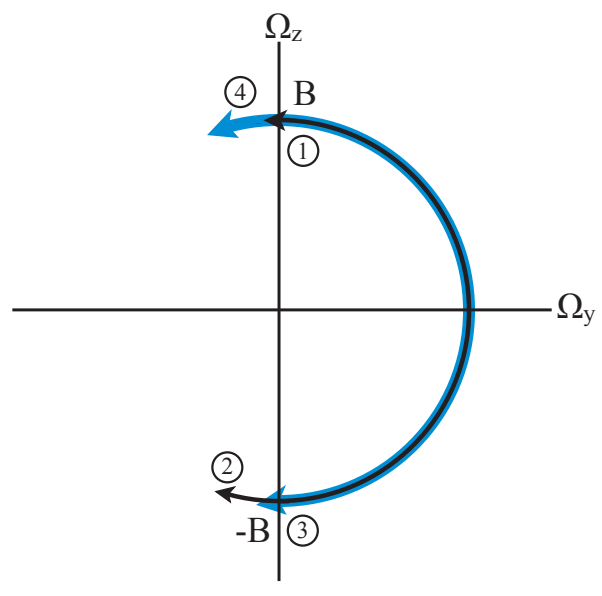

FIG. 3. Trajectory in the interaction frame for linearly modulated controls. The trajectory is the black path from 1-2 and back to 1 , followed by the blue path from 3-4 and back to 3 .

\section{Linear Modulation}

Returning to equation with $\Delta t=\frac{\pi}{A}$ and

$$
\begin{gathered}
\phi_{1}= \begin{cases}0 & 0<t \leq \Delta t \\
\pi & \Delta t<t \leq 3 \Delta t \\
0 & 3 \Delta t<t \leq 4 \Delta t\end{cases} \\
\dot{Y}=\left(A \epsilon \cos \phi_{1}(t) \Omega_{x}-\dot{\phi}_{2} \Omega_{z}\right) Y
\end{gathered}
$$

Moving into the interaction frame

$$
\begin{aligned}
Z \equiv \exp \left(-\int \epsilon A \cos \phi_{1}(t) d t \Omega_{x}\right) Y \\
\dot{Z}=-\dot{\phi}_{2}\left(\cos \epsilon \int A \cos \phi_{1}(t) d t \Omega_{z}\right. \\
\left.\quad+\sin \epsilon \int A \cos \phi_{1}(t) d t \Omega_{y}\right) Z
\end{aligned}
$$

We note that under the assumptions

$$
\int_{0}^{T} \cos \phi_{1}(t) d t=\int_{0}^{T} \dot{\phi}_{2}(t) d t=0
$$

the $\mathrm{Z}$ frame will agree with the $\mathrm{Y}$ frame which will agree with the lab frame at time $T$, so that it is sufficient to analyze the system in the interaction frame. Letting $\phi_{2}(t)$ be a linear modulation of the form

$$
\dot{\phi}_{2}= \begin{cases}-B & 0 \leq t<2 \Delta t \\ B & 2 \Delta t \leq t \leq 4 \Delta t\end{cases}
$$



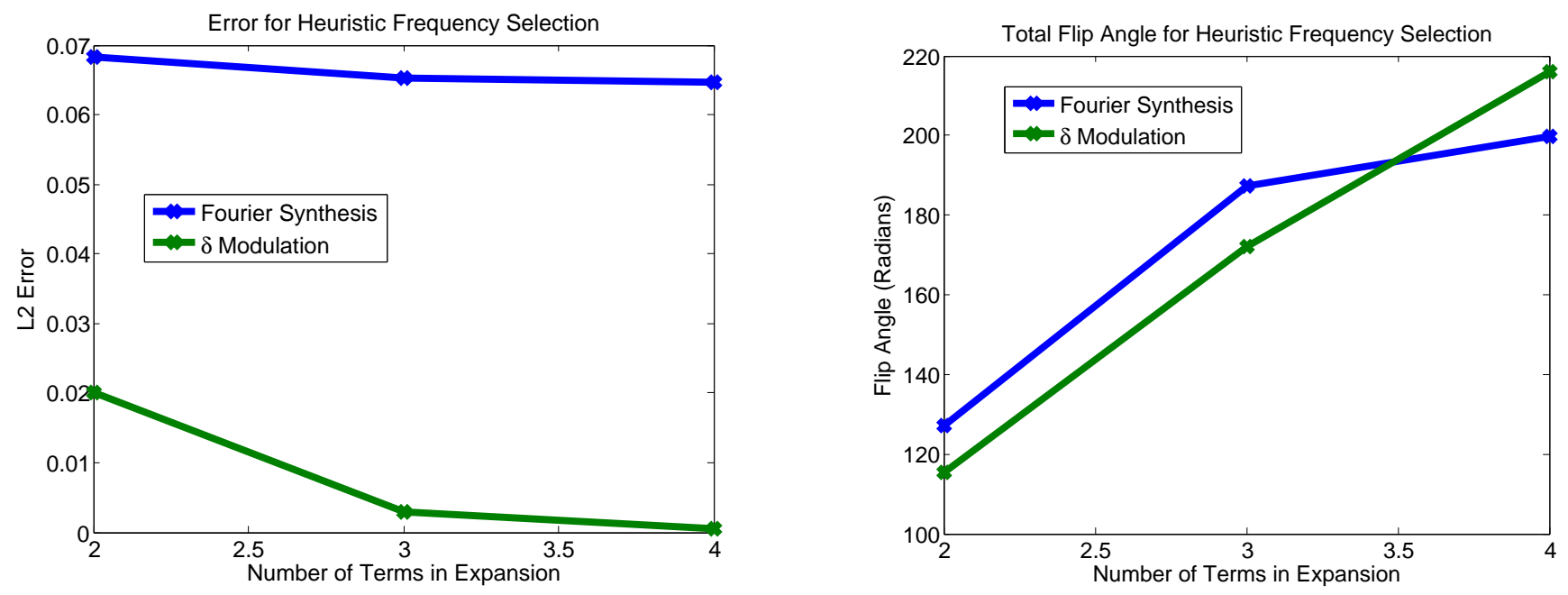

FIG. 4. L2 Error (Left) and Total Flip Angle (Right) for Heuristic Frequency Selection for Fourier Synthesis and $\delta$ Modulation. Error for a Fixed Pulse Duration is Smaller for $\delta$ Modulation.
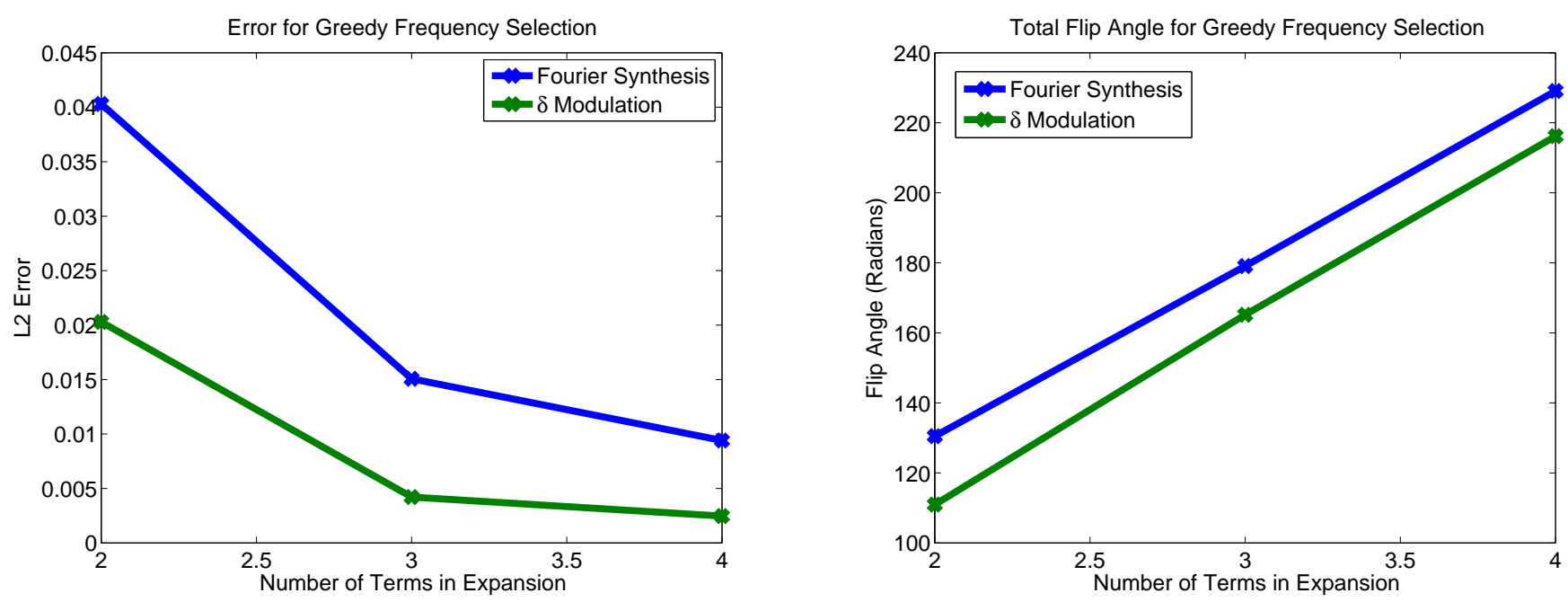

FIG. 5. L2 Error (Left) and Total Flip Angle (Right) for Greedy Frequency Selection for Fourier Synthesis and $\delta$ Modulation. Both Duration and Error are Smaller for $\delta$ Modulation.

we can analyze the resulting rotation with the PeanoBaker Series

$$
\begin{aligned}
\Phi & =I+\int_{0}^{\frac{4 \pi}{A}} H(t) d t+\int_{0}^{\frac{4 \pi}{A}} H(t) \int_{0}^{t} H\left(\sigma_{1}\right) d \sigma_{1} d t+\ldots \\
& =I+\int_{0}^{\frac{4 \pi}{A}} H(t) d t+o\left(\frac{B}{A}\right)^{2} \\
& =I+4 B \int_{0}^{\frac{\pi}{A}} \sin (A \epsilon t) d t \Omega_{y}+O\left(\frac{B}{A}\right)^{2} \\
& =I+\frac{4 B}{A \epsilon}(1-\cos (\pi \epsilon))+O\left(\frac{B}{A}\right)^{2} \\
& =I+\frac{8 B}{A}(1-\delta)+o(\delta)^{2}+O\left(\frac{B}{A}\right)^{2}
\end{aligned}
$$

which, to first order, has resulted in an evolution with the dispersion term reversed. Combining with a directly accessible evolution of $\frac{8 B(1+\delta)}{A} \Omega_{y}$ will produce a pulse that is robust to first order in $\delta$. Figure 3 displays the trajectory in the interaction frame for the linearly modulated pulse with $\epsilon>1$ and provides the intuition for why the dispersion term is negated.

\section{Arbitrary Modulation Schemes}

Other modulation functions are also possible. Let $|f(t)|<B$ be such a candidate modulation, then choos- 

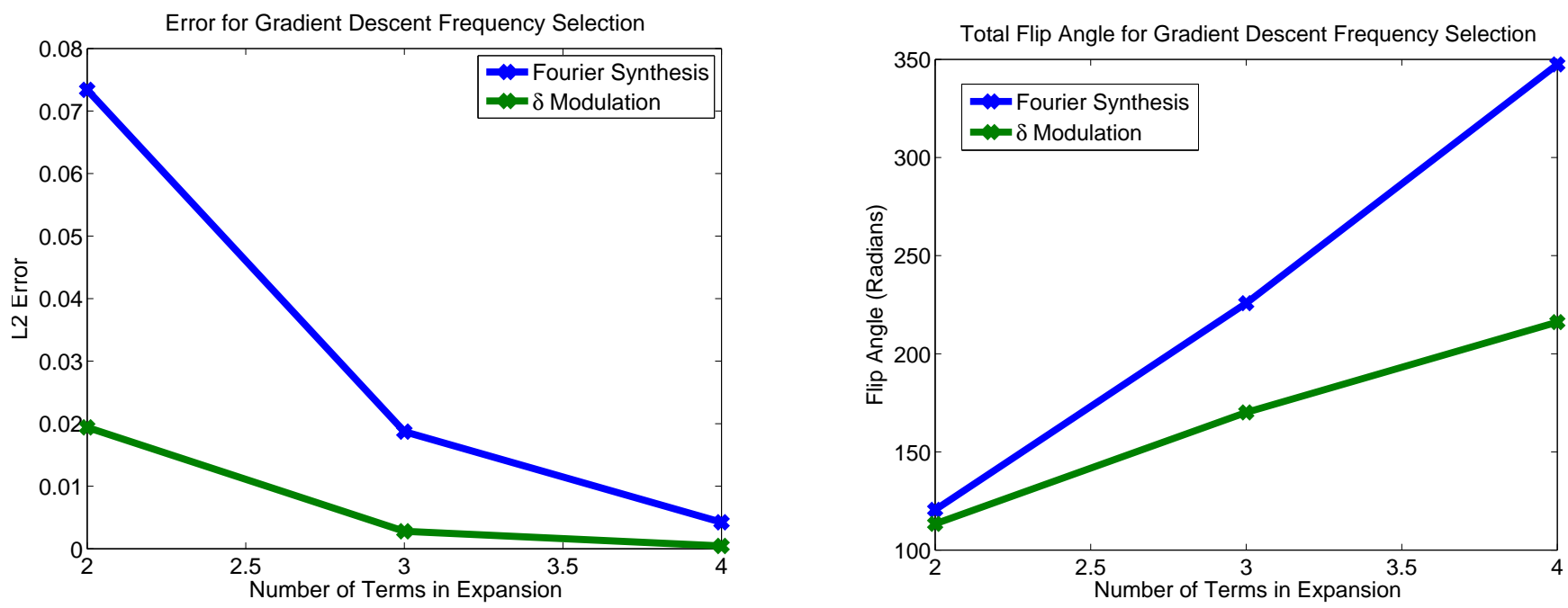

FIG. 6. L2 Error (Left) and Total Flip Angle (Right) for Gradient Descent Frequency Selection for Fourier Synthesis and $\delta$ Modulation. Both Duration and Error are Smaller for $\delta$ Modulation.

ing

$$
\dot{\phi}_{2}= \begin{cases}f(t) & 0<t \leq \Delta t \\ f(2 \Delta t-t) & \Delta t<t \leq 2 \Delta t \\ -f(t-2 \Delta t) & 2 \Delta t<t \leq 3 \Delta t \\ -f(4 \Delta t-t) & 3 \Delta t<t \leq 4 \Delta t\end{cases}
$$

will produce a rotation

$$
I-4 \int_{0}^{\frac{\pi}{A}} f(t) \sin (A \epsilon t) d t \Omega_{y}+O\left(\frac{B}{A}\right)^{2}
$$

which can be used to produce new dispersion dependencies and thereby robust pulses as was done in the linear case.

\section{CONCLUSION}

We have presented a new method for pulse design in the presence of RF-inhomogeniety that extends existing Fourier Synthesis Methods. The method displays superior time-error properties to conventional Fourier Synthesis Methods. This method is analytical and can be used to produce arbitrarily robust performance.
[1] B. Pryor and N. Khaneja, J. of Chem. Phys 194111, 125 (2006)

[2] M. H. Levitt, Prog. NMR Spectrosc. 18, 61 (1986).

[3] R. Tycko, Phys. Rev. Lett. 51 775(1983).

[4] R. Tycko, A. Pines, and J. Guckenheimer, J. Chem. Phys. 83, 2775(1985).

[5] A.J. Shaka, R. Freeman, J. Magn. Reson. 55, 487 (1983).

[6] M. Levitt and R. Freeman, J. Magn. Reson. 33, 473 (1979).

[7] M. Levitt and R.R. Ernst, J. Magn. Reson. 55, 247 (1983).

[8] M. Garwood, Y. Ke, J. Magn. Reson. 94, 511-525 (1991).

[9] N. Khaneja, C. Khelet, S.J. Glaser and N.C. Nielsen. J. Chem. Physics 124114503 (2006).

[10] T.E. Skinner, T. Reiss, B. Luy, N. Khaneja and S.J. Glaser, J. Magn. Reson., 163, 8, (2003).

[11] K. Kobzar, T.E. Skinner, N. Khaneja, S.J. Glaser and B. Luy, J. Magn. Reson., 170, 236, (2004).

[12] K. Kobzar, B. Luy, N. Khaneja, S.J. Glaser, J. Magn. Reson., 173, 229-235 (2005).
[13] K. Rohr and M. Speiss, Academic Press Inc, San Diego (1994).

[14] G. C. Chingas, A.N. Garroway, R. D. Bertrand, W. B. Moniz, J. Magn. Reson. 35, 283 (1979).

[15] M. S. Silver, R. I. Joseph, C.-N. Chen, V. J. Sank, D. I. Hoult, Nature. 310, 681 (1984).

[16] David E Rourke, Ph.D. Thesis (1992).

[17] M.Shinnar, S. Eleff, H. Subramanian, J. S. Leigh, Resonance Med. 12, 74-80 (1989).

[18] P. Le Roux, Proc. 7th SMRM 1049 (1988).

[19] S. Wimperis, J. Magn. Reson. B 109, 221 (1994).

[20] J. Jones, Phys. Rev. A. 67, 012317 (2002).

[21] K.R. Brown, A.W. Harrow, I.L. Chuang, Phys. Rev. A. 70, 052318 (2004).

[22] I. Roos and K. Moelmer, Phys. Rev. A. 69, 022321 (2004).

[23] M. Riebe, H. H äfner, C.F. Roos, W. Hänsel, J. Benhelm, G.P.T. Lancester, T.W. Körber, C. Bercher, F. SchmidtKaler, D.F.V. James, et al., Nature 429, 734 (2004).

[24] M. D. Barrett, J. Chiaverni, T. Schaetz, J. Britton, W.M. Itano, J.D. Jost, E. Knill, C. Langer, D. Leibfried, R. 
Ozeri, et. al., Nature 429, 737 (2004).

\section{PULSE PARAMETER APPENDIX}

Heuristic FSM, $\mathrm{n}=2$

$$
\begin{aligned}
\alpha= & {[187.3,33.8] } \\
\gamma= & {[49.3,196.5] } \\
\text { Pulse }: & {\left[(49.3)_{0}(4.5)_{90}(98.5)_{180}(4.5)_{90}(49.3)_{0}\right]^{\times 21}\left[(196.5)_{0}(4.2)_{90}(393.0)_{180}(4.2)_{90}(196.5)_{0}\right]^{\times 4} }
\end{aligned}
$$

Heuristic FSM, $\mathrm{n}=3$

$$
\begin{aligned}
\alpha & =[201.1,49.2,7.3] \\
\gamma & =[49.3,196.5,369.0]
\end{aligned}
$$

Pulse : $\quad\left[(49.3)_{0}(4.4)_{90}(98.5)_{180}(4.4)_{90}(49.3)_{0}\right]^{\times 23}$

$$
\left[(196.5)_{0}(4.1)_{90}(393.0)_{180}(4.1)_{90}(196.5)_{0}\right]^{\times 6}\left[(369.0)_{0}(3.6)_{90}(738.0)_{180}(3.6)_{90}(369.0)_{0}\right]^{\times 1}
$$

Heuristic FSM, n=4

$$
\begin{aligned}
\alpha & =[175.2903,18.3977,-10.8059,-5.67454] \\
\gamma & =[49.3,196.5,369.0,546.0]
\end{aligned}
$$

Pulse : $\quad\left[(49.3)_{0}(4.4)_{90}(98.5)_{180}(4.4)_{90}(49.3)_{0}\right]^{\times 20}\left[(196.5)_{0}(3.1)_{90}(393.0)_{180}(3.1)_{90}(196.5)_{0}\right]^{\times 3}$

$$
\left[(369.0)_{0}(-2.7)_{90}(738.0)_{180}(-2.7)_{90}(369.0)_{0}\right]^{\times 2}\left[(546.0)_{0}(-2.8)_{90}(1092.1)_{180}(-2.8)_{90}(546.0)_{0}\right]^{\times 1}
$$

Heuristic $\delta$ Mod, $\mathrm{n}=2$

$$
\begin{aligned}
\alpha= & {[105.5,16.7] } \\
\gamma= & {[90,270] } \\
\text { Pulse }: & {\left[(90.0)_{0}(180.0)_{175.6}(90.0)_{0}\right]^{\times 12}\left[(270.0)_{0}(540.0)_{175.8}(270.0)_{0}\right]^{\times 2} }
\end{aligned}
$$

Heuristic $\delta$ Mod, $\mathrm{n}=3$

$$
\begin{aligned}
\alpha= & {[108.3,22.4,4.3] } \\
\gamma= & {[90,270,450] } \\
\text { Pulse }: & {\left[(90.0)_{0}(180.0)_{175.8}(90.0)_{0}\right]^{\times 13}\left[(270.0)_{0}(540.0)_{176.3}(270.0)_{0}\right]^{\times 3}\left[(450.0)_{0}(900.0)_{177.9}(450.0)_{0}\right]^{\times 1} }
\end{aligned}
$$

Heuristic $\delta$ Mod, $\mathrm{n}=4$

$$
\begin{aligned}
\alpha= & {[109.8,25.7,7.1,1.2] } \\
\gamma= & {[90,270,450,630] } \\
\text { Pulse }: \quad & {\left[(90.0)_{0}(180.0)_{175.8}(90.0)_{0}\right]^{\times 13}\left[(270.0)_{0}(540.0)_{175.7}(270.0)_{0}\right]^{\times 3} } \\
& \quad\left[(450.0)_{0}(900.0)_{176.4}(450.0)_{0}\right]^{\times 1}\left[(630.0)_{0}(1260.0)_{179.4}(630.0)_{0}\right]^{\times 1}
\end{aligned}
$$

Greedy FSM, n=2

$$
\begin{aligned}
\alpha= & {[191.9,35.9] } \\
\gamma= & {[49.9,192.7] } \\
\text { Pulse }: & {\left[(49.9)_{0}(4.4)_{90}(99.9)_{180}(4.4)_{90}(49.9)_{0}\right]^{\times 22}\left[(192.7)_{0}(4.5)_{90}(385.4)_{180}(4.5)_{90}(192.7)_{0}\right]^{\times 4} }
\end{aligned}
$$

Greedy FSM, n=3

$$
\begin{aligned}
\alpha= & {[197.4,40.9,-3.8] } \\
\gamma= & {[49.9,192.7,502.9] } \\
\text { Pulse }: & {\left[(49.9)_{0}(4.5)_{90}(99.9)_{180}(4.5)_{90}(49.9)_{0}\right]^{\times 22}\left[(192.7)_{0}(4.1)_{90}(385.4)_{180}(4.1)_{90}(192.7)_{0}\right]^{\times 5} } \\
& \quad\left[(502.9)_{0}(-1.9)_{90}(1005.8)_{180}(-1.9)_{90}(502.9)_{0}\right]^{\times 1}
\end{aligned}
$$


Greedy FSM, n=4

$$
\begin{aligned}
\alpha & =[200.7,43.7,-5.9,-1.9] \\
\gamma & =[49.9,192.7,502.9,666.8]
\end{aligned}
$$

Pulse : $\quad\left[(49.9)_{0}(4.4)_{90}(99.9)_{180}(4.4)_{90}(49.9)_{0}\right]^{\times 23}\left[(192.7)_{0}(4.4)_{90}(385.4)_{180}(4.4)_{90}(192.7)_{0}\right]^{\times 5}$

$$
\left[(502.9)_{0}(-3.0)_{90}(1005.8)_{180}(-3.0)_{90}(502.9)_{0}\right]^{\times 1}\left[(666.8)_{0}(-0.9)_{90}(1333.7)_{180}(-0.9)_{90}(666.8)_{0}\right]^{\times 1}
$$

Greedy $\delta$ Mod, $\mathrm{n}=2$

$$
\begin{aligned}
\alpha= & {[105.5,16.6] } \\
\gamma= & {[86.7,259.1] } \\
\text { Pulse }: & {\left[(86.7)_{0}(173.4)_{175.6}(86.7)_{0}\right]^{\times 12}\left[(259.1)_{0}(518.1)_{175.8}(259.1)_{0}\right]^{\times 2} }
\end{aligned}
$$

Greedy $\delta$ Mod, $\mathrm{n}=3$

$$
\begin{aligned}
\alpha= & {[108.2,22.2,4.1] } \\
\gamma= & {[86.7,259.1,427.8] } \\
\text { Pulse : } & {\left[(86.7)_{0}(173.4)_{175.8}(86.7)_{0}\right]^{\times 13}\left[(259.1)_{0}(518.1)_{176.3}(259.1)_{0}\right]^{\times 3}\left[(427.8)_{0}(855.7)_{177.9}(427.8)_{0}\right]^{\times 1} }
\end{aligned}
$$

Greedy $\delta$ Mod, $\mathrm{n}=4$

$$
\begin{aligned}
\alpha= & {[108.5,22.9,4.6,-.3] } \\
\gamma= & {[86.7,259.1,427.8,730.2] } \\
\text { Pulse }: \quad & {\left[(86.7)_{0}(173.4)_{175.8}(86.7)_{0}\right]^{\times 13}\left[(259.1)_{0}(518.1)_{176.2}(259.1)_{0}\right]^{\times 3} } \\
& {\left[(427.8)_{0}(855.7)_{177.7}(427.8)_{0}\right]^{\times 1}\left[(730.2)_{0}(1460.5)_{180.2}(730.2)_{0}\right]^{\times 1} }
\end{aligned}
$$

Gradient Descent FSM, n=2

$$
\begin{aligned}
\alpha= & {[163.4,-15.7] } \\
\gamma= & {[51.5,373.7] } \\
\text { Pulse }: & {\left[(51.5)_{0}(4.3)_{90}(103.0)_{180}(4.3)_{90}(51.5)_{0}\right]^{\times 19}\left[(373.7)_{0}(-3.9)_{90}(747.4)_{180}(-3.9)_{90}(373.7)_{0}\right]^{\times 2} }
\end{aligned}
$$

Gradient Descent FSM, $\mathrm{n}=3$

$$
\begin{aligned}
\alpha= & {[169.6,-23.9,-10.3] } \\
\gamma=[52.4,379.1,550.3] & \\
\text { Pulse }: & {\left[(52.4)_{0}(4.5)_{90}(104.9)_{180}(4.5)_{90}(52.4)_{0}\right]^{\times 19}\left[(379.1)_{0}(-4.0)_{90}(758.2)_{180}(-4.0)_{90}(379.1)_{0}\right]^{\times 3} } \\
& {\left[(550.3)_{0}(-2.6)_{90}(1100.6)_{180}(-2.6)_{90}(550.3)_{0}\right]^{\times 2} }
\end{aligned}
$$

Gradient Descent FSM, n=4

$$
\begin{aligned}
\alpha= & {[174.4,-30.6,-19.0,-5.1] } \\
\gamma= & {[53.1,381.4,554.0,727.9] } \\
\text { Pulse }: & {\left[(53.1)_{0}(4.4)_{90}(106.1)_{180}(4.4)_{90}(53.1)_{0}\right]^{\times 20}\left[(381.4)_{0}(-3.8)_{90}(762.7)_{180}(-3.8)_{90}(381.4)_{0}\right]^{\times 4} } \\
& \quad\left[(554.0)_{0}(-3.2)_{90}(1108.0)_{180}(-3.2)_{90}(554.0)_{0}\right]^{\times 3}\left[(727.9)_{0}(-2.6)_{90}(1455.8)_{180}(-2.6)_{90}(727.9)_{0}\right]^{\times 1}
\end{aligned}
$$

Gradient Descent $\delta$ Mod, $\mathrm{n}=2$

$$
\begin{aligned}
& \alpha= {[105.5,16.6] } \\
& \gamma=[88.6,265.1] \\
& \text { Pulse: } \quad\left[(88.6)_{0}(177.1)_{175.6}(88.6)_{0}\right]^{\times 12}\left[(265.1)_{0}(530.1)_{175.9}(265.1)_{0}\right]^{\times 2}
\end{aligned}
$$

Gradient Descent $\delta$ Mod, $\mathrm{n}=3$

$$
\begin{aligned}
& \alpha=[108.3,22.4,4.3] \\
& \gamma=[89.1,267.0,444.5]
\end{aligned}
$$

Pulse : $\quad\left[(89.1)_{0}(178.1)_{175.8}(89.1)_{0}\right]^{\times 13}\left[(267.0)_{0}(534.1)_{176.3}(267.0)_{0}\right]^{\times 3}\left[(444.5)_{0}(889.0)_{177.9}(444.5)_{0}\right]^{\times 1}$ 
Gradient Descent $\delta$ Mod, $\mathrm{n}=4$

$$
\begin{aligned}
\alpha= & {[109.8,25.7,7.1,1.2] } \\
\gamma= & {[90.0,270.0,450.0,630] } \\
\text { Pulse }: \quad & {\left[(90.0)_{0}(180.0)_{175.8}(90.0)_{0}\right]^{\times 13}\left[(270.0)_{0}(540.0)_{175.7}(270.0)_{0}\right]^{\times 3} } \\
& {\left[(450.0)_{0}(900.0)_{176.4}(450.0)_{0}\right]^{\times 1}\left[(630.0)_{0}(1260.0)_{179.4}(630.0)_{0}\right]^{\times 1} }
\end{aligned}
$$

